EXTENDED REPORT

\title{
Ocular motor dysfunction and ptosis in ocular myasthenia gravis: effects of treatment
}

\section{J Kupersmith, G Ying}

See end of article for authors' affiliations

.....................

Correspondence to: Mark J Kupersmith, MD, Neuro-ophthalmology, Roosevelt Hospital, 1000 10th Avenue, New York, NY 10019, USA:

mkuper@bethisraelny.org

Accepted for publication 11 January 2005

\begin{abstract}
Aim: The optimal treatment of ocular myasthenia gravis (OMG) remains unknown. The authors evaluated the efficacy of prednisone and pyridostigmine in reducing diplopia, ocular motor dysfunction, and ptosis in patients with OMG.

Methods: Review of records from a clinical database from one neuro-ophthalmology service of patients presenting with OMG between 1990 and 2002, excluding those who developed generalised MG within the first month after diagnosis. Institutional review board approval was obtained for this study. Participants/interventions: Non-randomised, unmasked, therapy was given. 55 patients with diplopia in primary or downward gaze and clinically demonstrable extraocular muscle dysfunction received prednisone. 34 patients who had contraindications to steroids or who refused treatment with prednisone received pyridostigmine only. Over 5 days the daily prednisone dose was increased to 50-60 mg and then gradually reduced to $10 \mathrm{mg}$, followed by further reduction as tolerated. The pyridostigmine dose was begun at $180 \mathrm{mg}$ daily and increased as tolerated. Main outcome measures: Follow up evaluations, performed at 1,3-6, 12, and 24 months, detailed the frequency of ptosis and diplopia and the amount of ocular motor deviation in primary and downward gaze.

Results: The prednisone and pyridostigmine groups were similar for age, sex, acetylcholine receptor antibody level, prism cover test results for primary and downward gaze, diplopia in primary and downward gaze, and unilateral ptosis. Bilateral ptosis was present in $32.4 \%$ of the pyridostigmine group and $10.9 \%$ of the prednisone group $(p=0.02)$. The prednisone group showed resolution in primary gaze diplopia, downgaze diplopia, unilateral ptosis, and bilateral ptosis in $73.5 \%, 75.5 \%, 85.7 \%$, and $98 \%$, respectively at 1 month. The benefit persisted at 3-6, 12, and 24 months except for the bilateral ptosis. The pyridostigmine group showed resolution in primary gaze diplopia, downgaze diplopia, unilateral ptosis, and bilateral ptosis in $6.9 \%, 17.2 \%, 50 \%$, and $76.7 \%$ of patients after 1 month of treatment. The prism cover results improved $(p=0.003)$ in the prednisone group only. In the prednisone group, four patients had no response to therapy. Among the 51 prednisone responsive patients, there were 33 recurrences in 26 patients. 12 patients, all prednisone treated, had remissions. Except for three patients who developed diabetes, no patient developed a clinically significant systemic corticosteroid complication. Conclusion: These results suggest that $50-60 \mathrm{mg}$ daily prednisone followed by lower doses (10 mg or less) has the benefit of resolving ptosis and diplopia that lasts for at least 2 years in approximately $70 \%$ of patients.
\end{abstract}

METHODS

The charts of patients with OMG, evaluated by one neuroophthalmology Service (from 1990 through 2002) were reviewed and the data extracted for a confidential $\mathrm{OMG}$ database collected with consent from each patient. The age range was 4-82 years at the first examination when OMG was diagnosed (table $\mathrm{l}^{2}$ ). No patient had symptoms, such as swallowing, chewing, respiratory, or limb weakness or fatigue, or clinical examination findings of weakness in the muscles of the lower face or below the neck, suggestive of GMG. We excluded any patient with signs of a restrictive ocular myopathy or who developed GMG within a month of diagnosis as a result of use of a different treatment regimen.

Patients were not randomised for therapy. Patients with diplopia in primary or downward gaze and extraocular muscle dysfunction were offered prednisone therapy. Thirty four patients who refused or had contraindications to corticosteroid therapy, or who had only mild ptosis at the first examination were treated with pyridostigmine (pyridostigmine group). Five of the 55 patients in the prednisone group received pyridostigmine (mean dose of $120 \mathrm{mg}$ daily). Additionally, 10 patients initially treated with pyridostigmine, who did not improve, were later treated with

Abbreviations: GMG, generalised myasthenia gravis; $O M G$, ocular myasthenia gravis; PCT, prism cover test 
Table 1 Criteria for diagnosing ocular myasthenia gravis

(1) Ptosis in one or both upper lids not due to local lid disease, preferably that could fatigue or recover with rest.

(2) Extraocular muscle weakness in one or both eyes not in a strict third nerve muscle innervation pattern. If solely lateral rectus weakness,

clearcut fatiguability, recovery, or positive edrophonium test.

(3) Weakness can be present in one or both orbicularis oculi but no other weakness of the muscles of the head and neck.

(4) No pupillary abnormality other than from previous local disease or surgery.

(5) (A) plus (B), or (C) or (D) or (E) has to be present:

(A) Fatigue of the affected muscle with clear cut worsening of the ptosis after upward gaze for 30 to 60 seconds or worsening of the monocular duction after 120 seconds of gaze in the direction of action.

(B) Recovery of the upper lid ptosis to almost normal after 30 seconds to 10 minutes of eyelid closure. Recovery of the monocular duction after 120-180 seconds of gaze in the direction of the antagonist muscle.

(C) A positive edrophonium test

(D) Abnormal repetitive stimulation electromyography with a minimum decrement of $10 \%$.

(E) Abnormal serum acetylcholine receptor binding level.

prednisone. The medication dose was recorded at each visit, without other controls for compliance. No patient received other immunosuppressive agents. One patient in the prednisone group had a thymectomy for a thymoma, and was one of the patients who failed prednisone.

The prednisone dose, starting with $10 \mathrm{mg}$ daily, was escalated over 5 days to $50-60 \mathrm{mg}$ daily, and after 4 days the dose was reduced by $10 \mathrm{mg}$ per day each week until $10 \mathrm{mg}$ per day was achieved. The dose was further reduced by $2.5 \mathrm{mg}$ per day each week in response to controlling symptoms and findings of diplopia in primary gaze, difficulty reading, or ptosis that interfered with vision. Most patients in the prednisone group remained on a daily or alternate day dose of 2.5-10 mg. For analysis, alternate day prednisone was recorded as a daily dose that was half the alternate day dose (for example, a patient taking $10 \mathrm{mg}$ of prednisone every other day was considered to be taking $5 \mathrm{mg}$ a day). Recurrences were treated with reinstitution of half of the maximal dose originally given. All prednisone treated patients were prescribed a histamine receptor 2 blocking agent and calcium 1000-1500 mg and vitamin D 400-800 IU daily.

The pyridostigmine group started therapy with $180 \mathrm{mg}$ daily in divided doses and received higher doses until the ocular symptoms did not interfere with vision function or side effects developed despite medical co-interventions. The mean maximum daily dose was $272 \mathrm{mg}$ (median $240 \mathrm{mg}$; range $180-720 \mathrm{mg}$ ) and 24 patients received at least $240 \mathrm{mg}$ per day.

In the prednisone group (including 10 patients switched from the pyridostigmine group), a recurrence was defined as the return of resolved diplopia in primary or downward gaze, as the dose of prednisone was reduced, and failed to resolve with an increase of $10 \mathrm{mg}$ daily. The time from reaching a daily dose of $10 \mathrm{mg}$ of prednisone before a recurrence and the daily dose of prednisone when the recurrence occurred were recorded. A treatment failure was defined as persistent diplopia in primary or downward gaze or ptosis that covered either pupil with the eyes in primary gaze despite higher prednisone doses or if $20 \mathrm{mg}$ of daily prednisone was needed. A remission was defined as having no ptosis, no diplopia, and normal ocular muscle ductions after stopping therapy for at least 6 months.

The baseline examination was performed when treatment was prescribed. At each visit patients were questioned whether diplopia was present in primary or downward gaze, particularly while reading or descending stairs over the previous week. All examinations detailed the presence of ptosis and prism cover test (PCT) measurement (in dioptres) for distance primary and downward gaze. To account for variability with worsening of the findings typically observed after repeated testing in untreated patients, only initial measurements of ptosis and the cover test were recorded Patients were evaluated at each visit for GMG and in prednisone treated patients, for corticosteroid complications.

\section{Analysis (using SAS 8.2)}

The descriptive analysis was performed for the baseline characteristics and longitudinal outcome measurements. For subsequent examinations, we evaluated whether the diplopia in primary or downward gaze or the ptosis in one or both eyes persisted, developed, or resolved, and if the PCT measurements were reduced. The PCT measurements at baseline were compared with the measurements at each subsequent examination. We devised a calculation, which considers that in some patients the vertical and horizontal PCT measurements might worsen or improve independently of each other. We computed a vector determination composed of both the horizontal and vertical measurements of the PCT measurement for each direction of gaze.

The comparison of baseline characteristics between two treatment groups was performed using $\chi^{2}$ test for the categorical measurements and Wilcoxon rank sum test for the continuous measurements because of the skewed distributions. For each treatment, the efficacy was evaluated by the comparison of measurements from follow up with those from baseline using the generalised equation estimate to adjust the correlation from the longitudinal repeated measurements. ${ }^{11}$ The paired $t$ test was used to evaluate the baseline and 1 month vector calculations of the PCT results. The efficacy difference between two treatments was assessed using the generalised equation estimate with the adjustment of baseline characteristics. For the prednisone group, the effect of baseline characteristics on the treatment failure, remission and recurrence was examined using the linear regression. For the prednisone group, the treatment failure, no response, remission, and recurrence data were considered with and without the data for the 10 patients switched to prednisone after failing pyridostigmine.

\section{RESULTS}

The baseline features (table 2) were comparable between the groups except the pyridostigmine group had more frequent bilateral ptosis $(\mathrm{p}=0.02)$.

Compared with the baseline evaluation, there was a significant frequency of resolution of ptosis and diplopia and improvement in the PCT measurements in the prednisone group (table 3 ). The pyridostigmine group did not show the same frequency of diplopia resolution or improvement of PCT measurements at 1 month and 3-6 months. Except for bilateral ptosis, the prednisone group had significant benefit for all features that persisted at 3-6, 12, and 24 months. The prednisone group was significantly better than the pyridostigmine group at the 1 month and 3-6 month evaluations (table 3). The prednisone group had significantly better PCT measurements at 1 month and 36 months except for the vertical prism measurement in primary gaze at 3-6 months (table 4 ). The patients in the prednisone group had significant reduction in the mean vector measurement comparing baseline and 1 month, for primary gaze $(15.9$, SD 11.1 and 4.2, SD 8.1, p=0.001) and for downward gaze (12.8, SD 8.6 and 3.3, SD 7.4, p=0.001). The pyridostigmine group did not show the same improvement in the vector measurements for baseline and 1 month 
Table 2 Baseline characteristics of patients in prednisone and pyridostigmine treatment groups

\begin{tabular}{|c|c|c|c|}
\hline Characteristics & $\begin{array}{l}\text { Prednisone group } \\
(\mathrm{n}=55)\end{array}$ & $\begin{array}{l}\text { Pyridostigmine } \\
\text { group }(n=34)\end{array}$ & \\
\hline Abnormal acetylcholine receptor antibody & $\begin{array}{l}(n=26) \\
\text { Mean (SD) }\end{array}$ & $\begin{array}{l}(n=15) \\
\text { Mean (SD) }\end{array}$ & p Value* \\
\hline Age (years) & $48.4(15.5)$ & $52.6(23.8)$ & 0.16 \\
\hline Acetylcholine receptor antibody $(\mathrm{nmol} / \mathrm{l})$ & $2.7(9.6)$ & $2.8(6.4)$ & 0.35 \\
\hline Primary gaze, horizontal PCT (dioptres) & $12.2(12.1)$ & $13.3(10.8)$ & 0.54 \\
\hline Primary gaze, vertical PCT (dioptres) & $5.5(6.3)$ & $5.4(6.4)$ & 0.84 \\
\hline Downward gaze, horizontal PCT (dioptres) & $9.8(10.4)$ & $11.1(9.5)$ & 0.44 \\
\hline Downward gaze, vertical PCT (dioptres) & $\begin{array}{l}6.3(6.2) \\
\text { n (\%) }\end{array}$ & $\begin{array}{l}5.5(6.9) \\
\text { n (\%) }\end{array}$ & $\begin{array}{l}0.50 \\
\text { p Valuet }\end{array}$ \\
\hline Sex-male & $30(54.6 \%)$ & $17(50.0 \%)$ & 0.83 \\
\hline Abnormal acetylcholine receptor antibody & $26(47.2 \%)$ & $17(50 \%)$ & 0.71 \\
\hline Diplopia primary gaze & $52(94.6 \%)$ & $29(85.3 \%)$ & 0.25 \\
\hline Diplopia downward gaze & $45(81.8 \%)$ & $27(79.4 \%)$ & 0.79 \\
\hline Ptosis, unilateral & $25(45.5 \%)$ & $17(50.0 \%)$ & 0.83 \\
\hline Ptosis, bilateral & $6(10.9 \%)$ & $11(32.4 \%)$ & 0.02 \\
\hline
\end{tabular}

follow up primary gaze (17.9, SD 10.5 and 16.5, SD 10.4) or downward gaze (14.7, SD 8.5 and 13.3, SD 7.8).

In patients treated with prednisone, at 12 months, the diplopia resolved in five of 10 patients with diplopia at 3-6 months and at 24 months, in five of 14 patients with diplopia at 12 months. Of the patients withdrawn from prednisone, diplopia was absent in 10/17 at 12 months and in $10 / 16$ at 24 months.

Of the 61 patients who responded to prednisone, there were 43 recurrences in 31 patients occurring at a mean of 5.2 months after the prednisone was reduced to $10 \mathrm{mg}$ daily (table 5). There were eight failures in the prednisone group, four who never resolved their diplopia and four who had a recurrence as the dose was reduced below $20 \mathrm{mg}$ daily; 12 prednisone treated patients (18.5\%) had remissions. Age, sex, abnormal acetylcholine receptor antibody level, ptosis, and diplopia in primary or downward gaze at baseline were not correlated with treatment failure or the number of remissions or recurrences.

Two patients in the pyridostigmine group had the diplopia resolve at 1 month. The diplopia recurred in one of the two by 6 months. Because of the onset of diplopia in two additional patients at 1 month, the percentage of patients with diplopia was the same as at baseline when accounting for missing data. No patient in the pyridostigmine group had a remission.
The 12 month and 24 month results for the pyridostigmine group are not reported owing to a large drop out rate.

No patient developed a clinically apparent fracture, new onset systemic hypertension, or an infection that required hospitalisation. Three of 65 patients treated with prednisone developed new onset diabetes mellitus.

\section{DISCUSSION}

In the present study, less than $10 \%$ of patients had ptosis only, less than $30 \%$ had diplopia only, while the majority (64\%) had both diplopia and ptosis, which differs from OMG in children where ptosis is frequent and diplopia is uncommon. ${ }^{12}$ Combined horizontal and vertical ocular misalignment was most frequent (43.5\%) but horizontal (34.1\%) or vertical $(22.4 \%)$ deviations alone occurred.

Our results confirm the findings of a previous study that moderate dose (50-60 mg) daily prednisone, reduced over 6 weeks, followed by $10 \mathrm{mg}$ or less daily, resolves diplopia in primary and downward gaze more frequently than with pyridostigmine alone. This treatment regimen also reduces the amount of PCT measured ocular deviation in primary and downward gaze. The immediate and long term benefit also occurred in patients who failed pyridostigmine alone. Prednisone decreased the frequency of ptosis in one or both upper lids from $56.4 \%$ to $16.3 \%$ at 1 month and to $15.8 \%$ at

Table 3 Comparison of treatment effect on outcome measurements for baseline groups for categorical outcome measurements

\begin{tabular}{|c|c|c|c|c|c|c|c|c|c|c|c|c|}
\hline \multirow{2}{*}{$\begin{array}{l}\text { Yes (\%) } \\
\text { Times } \\
\text { (months) }\end{array}$} & \multicolumn{2}{|c|}{$\begin{array}{l}\text { Treatment } \\
\text { Mean daily dose } \\
\text { (mg) }\end{array}$} & \multicolumn{2}{|c|}{$\begin{array}{l}\text { Follow up } \\
\text { completed }\end{array}$} & \multicolumn{2}{|c|}{$\begin{array}{l}\text { Diplopia primary } \\
\text { gaze } \\
\text { No of patients (\%) }\end{array}$} & \multicolumn{2}{|c|}{$\frac{\text { Diplopia downgaze }}{\text { No of patients (\%) }}$} & \multicolumn{2}{|c|}{$\begin{array}{l}\text { Ptosis, unilateral } \\
\text { No of patients (\%) }\end{array}$} & \multicolumn{2}{|c|}{$\frac{\text { Ptosis, bilateral }}{\text { No of patients (\%) }}$} \\
\hline & Pred & Pyrid & Pred & Pyrid & Pred & Pyrid & Pred & Pyrid & Pred & Pyrid & Pred & Pyrido \\
\hline 0 & & & 55 & 34 & $52(94.6)$ & $29(85.3)$ & $45(81.8)$ & $27(79.4)$ & $25(45.5)$ & $17(50.0)$ & $6(10.9)$ & $11(32.4)$ \\
\hline 1 & 13 & 272 & 49 & 30 & $13(26.5)$ & $27(93.1)$ & $12(24.5)$ & $24(82.8)$ & 7 (14.3) & $15(50.0)$ & $1(2.0)$ & $7(23.3)$ \\
\hline $3-6$ & 5 & 323 & 50 & 20 & $13(26.0)$ & $16(76.2)$ & $15(30.0)$ & 15 (71.4) & $8(16.0)$ & $8(40.0)$ & $1(2.0)$ & $7(35.0)$ \\
\hline 12 & 2.5 & 308 & 41 & 5 & $12(28.6)$ & $7(100)^{*}$ & $10(23.8)$ & $7(100)^{*}$ & 8 (19.5) & $4(57)^{*}$ & $1(2.4)$ & $2(28.5)^{*}$ \\
\hline 24 & 3.0 & 330 & 38 & 6 & $11(29.0)$ & $4(67)^{*}$ & $11(29.7)$ & $4(67)^{*}$ & $4(10.5)$ & $1(16.7)^{*}$ & $2(5.3)$ & $2(33.3)^{*}$ \\
\hline $\mathrm{p}$ Valuet & & & & & $<0.0001$ & 0.20 & $<0.0001$ & 0.55 & 0.002 & 0.62 & 0.07 & 0.22 \\
\hline p Value & & & & & $<0.0001$ & & 0.0001 & & 0.004 & & 0.04 & \\
\hline
\end{tabular}

Pred, prednisone; Pyrid, pyridostigmine.

*No or insufficient data available for analysis

†For the comparison of outcome measure at follow up with that at baseline within the same treatment group

fFor the comparison of the outcome measure at follow up of 1 month and 3-6 months between the prednisone and pyridostigmine groups after adjustment for baseline level, age, sex, and antibody level. 
Table 4 Comparison of treatment effect on outcome measurements for baseline groups for continuous outcome measurements (prism cover test)

\begin{tabular}{|c|c|c|c|c|c|c|c|c|c|c|}
\hline \multirow{3}{*}{$\begin{array}{l}\text { Mean (SD) } \\
\text { Times } \\
\text { (months) }\end{array}$} & \multirow{2}{*}{\multicolumn{2}{|c|}{$\begin{array}{l}\text { Follow up } \\
\text { completed }\end{array}$}} & \multirow{2}{*}{\multicolumn{2}{|c|}{$\begin{array}{l}\text { Primary gaze, } \\
\text { horizontal } \\
\text { No of dioptres (SD) }\end{array}$}} & \multirow{2}{*}{\multicolumn{2}{|c|}{$\begin{array}{l}\text { Primary gaze, } \\
\text { vertical } \\
\text { No of dioptres (SD) }\end{array}$}} & \multirow{2}{*}{\multicolumn{2}{|c|}{$\begin{array}{l}\text { Downward gaze, } \\
\text { horizontal } \\
\text { No of dioptres (SD) }\end{array}$}} & \multirow{2}{*}{\multicolumn{2}{|c|}{$\begin{array}{l}\text { Downward gaze, } \\
\text { vertical } \\
\text { No of dioptres (SD) }\end{array}$}} \\
\hline & & & & & & & & & & \\
\hline & Pred & Pyrid & Pred & Pyrid & Pred & Pyrid & Pred & Pyrid & Pred & Pyrid \\
\hline 0 & 55 & 34 & $12.2(12.1)$ & $13.3(10.8)$ & $5.5(6.2)$ & $5.4(6.4)$ & $9.8(10.4)$ & $11.1(9.5)$ & $6.3(6.2)$ & $5.5(6.9)$ \\
\hline 1 & 49 & 30 & $4.1(8.3)$ & 13.0 (12.5) & $0.8(2.0)$ & $5.0(6.7)$ & $2.1(6.0)$ & $10.9(11.6)$ & $1.1(3.7)$ & $4.6(5.4)$ \\
\hline $3-6$ & 50 & 20 & $5.3(9.4)$ & $6.2(5.9)$ & $3.2(6.8)$ & $3.0(5.6)$ & $2.8(6.6)$ & $8.9(9.5)$ & $1.6(3.3)$ & 4.2 (6.7) \\
\hline 12 & 41 & 5 & $5.2(11.0)$ & $22(9.3)^{*}$ & $1.3(3.9)$ & $25^{\star}$ & $3.2(8.3)$ & $17.7(15)^{*}$ & 1.7 (4.6) & $12^{*}$ \\
\hline 24 & 38 & 6 & $3.7(8.1)$ & * & 1.1 (2.9) & * & $2.9(7.9)$ & * & $0.6(2.6)$ & * \\
\hline p Valuet & & & 0.003 & 0.12 & 0.0008 & 0.25 & 0.008 & 0.73 & 0.006 & 0.71 \\
\hline p Valuef & & & 0.007 & & 0.11 & & 0.01 & & 0.02 & \\
\hline
\end{tabular}

Pred, prednisone; Pyrid, pyridostigmine.

*No or insufficient data available for analysis

†For the comparison of outcome measure at follow-up with that at baseline within the same treatment group

tFor the comparison of the outcome measure at follow up of 1 month and 3-6 months between the prednisone and pyridostigmine groups after adjustment for baseline level, age, sex, and antibody level.

2 years. Our results contrast with those reported for childhood OMG where only $29 \%$ of 24 patients treated with unspecified doses of prednisone, pyridostigmine, or both for an unspecified time improved ocular motility. ${ }^{12}$ In the current study, the frequency of maintaining improved ocular motor and lid function might have been higher if the prednisone dose was reduced more slowly, as is recommended for controlling systemic muscle weakness. ${ }^{13}$ We reduced the prednisone dose quickly to avoid adverse effects. Some benefit was probably gained by the combination of pyridostigmine and prednisone.

Our failure rate of $12.3 \%$ contrasts with Evoli's study where $6 / 18(33 \%)$ failed to improve ocular motor function with prednisone $25-50 \mathrm{mg}$ alternate day therapy. ${ }^{7}$ In fact, of our eight failures, only four $(6.1 \%)$ failed to improve with moderate doses. Although azathioprine appears to be effective, it can take 6 months to improve the ocular motor dysfunction and is less practical for patients needing a rapid response. ${ }^{14}$ Also, azathioprine (mean dose $145 \mathrm{mg}$ ) improved, but did not necessarily normalise, the OMG in 19 of 22 patients. ${ }^{14}$ Azathioprine or other immunosuppressive agents could be considered for patients who fail prednisone. Generally, thymectomy, in patients without a thymoma, has not been as the primary treatment of OMG, although reports suggest it is effective. ${ }^{1516}$ As new safer surgical approaches evolve, thymectomy may be investigated in OMG. ${ }^{17}$

The remission frequency was $18.5 \%$ for all patients treated with prednisone and zero for patients treated with pyridostigmine alone. The remission rate in the pyridostigmine group could have been artificially low owing to poor follow up. Remission rates of $11 \%$ in 108 patients ${ }^{4}$ and $30 \%$ in 43 patients $^{2}$ with OMG who did not generalise have been described, without reference to treatment.

A review of OMG treatment emphasised the complications of rate of chronic corticosteroid use, ${ }^{18}$ but chronic low dose daily or alternate day prednisone, particularly with cointerventions to prevent complications, may not result in frequent severe adverse effects. ${ }^{19}{ }^{20}$ Our prednisone treated patients had no uncontrollable corticosteroid complications, possibly due to the effort to reduce the dose, not to use high maintenance doses, to accept a degree of ocular symptoms, and not to continue prednisone in patients who failed therapy, and the use of daily calcium and vitamin D. There were no clinically symptomatic hip or lumbar fractures.

In our pyridostigmine group, only two of 34 patients with diplopia had the diplopia resolve and one of these recurred. This contrasts with studies where pyridostigmine markedly improved ocular signs of OMG in $9 / 13$ patients but the definition of "improved" was unspecified. ${ }^{7}$ In another report, $50 \%$ of 14 patients treated with pyridostigmine, approximately $150 \mathrm{mg}$ per day, had "considerable improvement," but this term was also not defined. ${ }^{17}$ In the current study, after 1 month of pyridostigmine, 50\% of patients had ptosis of an upper lid, similar to the baseline frequency, but approximately one third of patients with bilateral ptosis had resolution of the ptosis. The pyridostigmine treatment results might have been better but side effects precluded using

\begin{tabular}{|c|c|c|c|c|}
\hline Outcome & $\begin{array}{l}\text { Patients initially } \\
\text { treated with } \\
\text { prednisone }(n=55)\end{array}$ & $\%$ of 55 & $\begin{array}{l}\text { Patients treated } \\
\text { with prednisone } \\
\text { any time }(n=65)\end{array}$ & $\%$ of 65 \\
\hline Treatment failure & 7 & 12.7 & 8 & 12.3 \\
\hline Remission & 11 & 20.0 & 12 & 18.5 \\
\hline Unknown & 1 & 1.8 & 1 & 1.5 \\
\hline Never responded & 4 & 7.3 & 4 & 6.2 \\
\hline $\begin{array}{l}\text { Number of recurrences } \\
\text { among responder }\end{array}$ & & & & \\
\hline 0 & 23 & 41.8 & 28 & 43.1 \\
\hline 1 & 21 & 38.2 & 23 & 35.4 \\
\hline 2 & 4 & 7.3 & 6 & 9.2 \\
\hline 4 & 1 & 1.8 & 2 & 3.1 \\
\hline
\end{tabular}

Two patients (3.6\%) had incomplete or missing data. Age, sex, antibody level, ptosis at baseline were all not significantly associated with any above outcomes. 
higher doses and we had limited follow up at 12 months and 24 months, possibly because of treatment failure. Our conclusions are biased towards prednisone treatment since our goal was to restore visual function as rapidly as possible.

Prednisone and pyridostigmine for OMG are not truly comparable therapies since prednisone alters the autoimmune disease process and pyridostigmine does not. It appears that prednisone, given judiciously, is a reasonable therapy for restoring visual function in OMG. Long term follow up with measures of patient visual function and safety documentation of chronic low dose prednisone therapy are needed before final recommendations can be made.

\section{Authors' affiliations}

M J Kupersmith, Neuro-ophthalmology Service of Roosevelt Hospital, and New York Eye and Ear Infirmary, New York, NY, USA

G-S Ying, University of Pennsylvania School of Medicine, Philadelphia, PA, USA

Supported by the Michael Fischer Foundation.

\section{REFERENCES}

1 Osserman K. Ocular myasthenia gravis. Invest Ophthalmol Vis Sci 1967;6:277-86.

2 Oosterhuis $\mathrm{H}$. The ocular signs and symptoms of myasthenia gravis. Doc Ophthalmol 1982;52:363-78.

3 Schlezinger N, Fairfax W. Evaluation of ocular signs and symptoms in myasthenia gravis. Arch Ophthalmol 1959;62:985-90.

4 Bever C, Aquino A, Penn A, et al. Prognosis of ocular myasthenia. Ann Neurol 1983;14:516-19.
5 Oosterhuis $\mathbf{H}$. The natural course of myasthenia gravis: a long term follow up study. J Neurol Neurosurg Psychiatry 1989;52:1121-7.

6 Grob D. Natural history of myasthenia gravis. In Engel AG, eds. Myasthenia gravis and myasthenic disorders. Oxford: Oxford University Press, 1999:135-6.

7 Evoli A, Tonali P, Bartoccioni E, et al. Ocular myasthenia: diagnostic and therapeutic problems. Acta Neurol Scand 1988;77:31-5.

8 Fischer K, Schwartzman R. Oral corticosteroids in the treatment of ocular myasthenia gravis. Ann NY Acad Sci 1976;274:652-8.

9 Kupersmith MJ, Moster M, Bhuiyan S, et al. Beneficial effects of corticosteroids on ocular myasthenia gravis. Arch Neurol 1996;53:802-4.

10 Kupersmith MJ, Latkany R, Homel P. Development of generalized disease at 2 years in patients with ocular myasthenia gravis. Arch Neurol 2003;60:243-8.

11 Liang, KY, Zeger SL. Longitudinal data analysis using generalized linear models. Biometrika 1986;73:13-22.

12 Kim JH, Hwang JM, Hwang Y, et al. Childhood ocular myasthenia gravis. Ophthalmology 2003;110:1458-62.

13 Mann JD, Johns TR, Campa JF. Long-term administration of corticosteroids in myasthenia gravis. Neurology 1991;26:729-40.

14 Sommer N, Sigg B, Melms A, et al. Ocular myasthenia gravis: response to long term immunosuppressive treatment. I Neurol Neurosurg Psychiatry 1997;62:156-62.

15 Schumm M, Wietholter $\mathrm{H}$, Fateh-Moghadam A, et al. Thymectomy in myasthenia with pure ocular symptoms. I Neurol Neurosurg Psychiatry 1985;48:332-7.

16 Nakamura H, Taniguchi Y, Suzuki Y, et al. Delayed remission after thymectomy for myasthenia gravis for the purely ocular type. J Thorac Cardiovasc Surg 1996:112:371-6.

17 Shrager J, Deep M, Mick R, et al. Transcervical thymectomy for myasthenia gravis achieves results comparable to thymectomy by sternotomy. Ann Thorac Surg 2002;74:320-6.

18 Kaminski H, Daroff R. Treatment of ocular myasthenia. Steroids only when compelled. Arch Neurol 2000;57:752-3.

19 Caldwell J, Furst D. The efficacy and safety of low-dose corticosteroids for rheumatoid arthritis. Sem Arth Rheum 1991;21:1-11.

20 Spiera RF, Mitnick HJ, Kupersmith MJ, et al. A prospective double-blind randomized placebo-controlled trial of methotrexate in the treatment of giant cell arteritis (GCA). Clin Experimental Rheumatol 2001;19:495-501. 